\title{
Outcomes of slide tracheoplasty in 101 children: A 17-year single-center experience
}

\author{
Colin R. Butler, MRCS, Simone Speggiorin, MD, Friso M. Rijnberg, BSc, Derek J. Roebuck, FRCR, \\ Nagarajan Muthialu, FRCS, Richard J. Hewitt, FRCS, and Martin J. Elliott, MD, FRCS
}

\begin{abstract}
Objective: Our study describes the results from surgical slide tracheoplasty (STP) in children with long segment tracheal stenosis.
\end{abstract}

\begin{abstract}
Methods: Demographic and preoperative conditions, operative details, and outcome measures, including the need for endoscopic airway intervention and mortality, were collected for children undergoing STP between February 1995 and December 2012.
\end{abstract}

\begin{abstract}
Results: One hundred one patients (median age, 5.8 months; range, 5 days-15 years) underwent STP. Seventy-two patients $(71.3 \%)$ had associated cardiovascular anomalies. Preoperative ventilation was necessary in 56 patients $(55.4 \%)$, whereas extracorporeal membrane oxygenation was required in 10 patients $(9.9 \%)$. Abnormal bronchial arborization was present in 39 children $(38.6 \%)$, which included 13 patients $(12.8 \%)$ with an anomalous right upper lobe bronchus and 17 patients $(16.8 \%)$ with tracheal trifurcation. Airway stenosis extended into 1 or both bronchi in 24 patients $(23.7 \%)$ and preoperative malacia was present in 24 patients $(23.7 \%)$ ). STP was extended into the bronchus in 47 patients $(46.5 \%)$ ). Overall survival was $88.2 \%$ (mortality in 12 patients). Post-STP balloon dilation was necessary in 45 patients $(44.6 \%)$ and stenting was required in 22 patients $(21.8 \%)$. Multivariate analysis revealed preoperative extracorporeal membrane oxygenation $(P<.05)$, preoperative malacia $(P<.001)$, and bronchial stenosis $(P<.05)$ to be adverse predictors of survival. Preoperative malacia was a significant risk factor for stenting $(P<.05)$.

Conclusions: STP is a versatile and reliable technique associated with low morbidity and mortality when compared with previous strategies for children with long segment tracheal stenosis. The presence of preoperative bronchomalacia is a significant risk factor for death and postoperative stenting. (J Thorac Cardiovasc Surg 2014;147:1783-90)
\end{abstract}

Long segment congenital tracheal stenosis (LSCTS) is a severe and often life-threatening anatomical anomaly constricting the trachea for $>50 \%$ of its length. Whereas a defining feature is the presence of complete tracheal rings, many patients have other congenital anomalies. Cardiovascular malformations such as pulmonary artery sling and ventricular septal defects are common; however, both pulmonary and gastrointestinal defects may also be present, highlighting the heterogeneity found in these groups of children. ${ }^{1,2}$

Historically, LSCTS has often been associated with significant morbidity and mortality with the various surgical

\footnotetext{
From the National Service for Severe Tracheal Disease in Children, Great Ormond Street Hospital for Children, National Health Service Foundation Trust, London, United Kingdom.

Disclosures: Authors have nothing to disclose with regard to commercial support.

Read at the 93rd Annual Meeting of The American Association for Thoracic Surgery, Minneapolis, Minnesota, May 4-8, 2013.

Received for publication Aug 13, 2013; revisions received Feb 18, 2014; accepted for publication Feb 25, 2014; available ahead of print April 1, 2014.

Address for reprints: Colin R. Butler, MRCS, The National Service for Severe Tracheal Disease in Children, Great Ormond Street Hospital for Children, National Health Service Foundation Trust, London, WC1N 3JH, United Kingdom (E-mail: colin.butler@gosh.nhs.uk).

$0022-5223 / \$ 36.00$

Copyright (C) 2014 by The American Association for Thoracic Surgery

http://dx.doi.org/10.1016/j.jtcvs.2014.02.069
}

approaches advocated. ${ }^{3-8}$ These have included simple resection, augmentation with cartilage or pericardial patch graft, replacement with homograft, or slide tracheoplasty (STP) ${ }^{9,10}$ Following initial reports from centers where STP was shown to have a favorable outcome, we became early adopters of this technique., ${ }^{71-13}$ Despite its increasing use, most reported studies have been small and long-term outcomes are rarely reported.

As the nationally funded quaternary referral unit for complex pediatric airway disease, our unit receives nearly all new cases of LSCTS in the United Kingdom. Our approach to management involves a dedicated multidisciplinary team, ${ }^{13}$ and we now have gained $>17$ years of experience in managing a diverse group of children with very complex tracheal disease. This study reviews our results and endeavors to determine predictors of adverse outcome for children with LSCTS undergoing STP.

\section{MATERIALS AND METHODS}

Our study was a retrospective, consecutive case series of children undergoing STP for LSCTS at Great Ormond Street Hospital for Children, London, between February 1995 and December 2012. Institutional review board approval was granted for this study. Data were recorded in a registered database and subsequently analyzed for demographic and specific pre- and perioperative characteristics. These included the presence of cardiac and noncardiac anomalies, minimal tracheal diameter, airway 


\section{Abbreviations and Acronyms \\ $\mathrm{CPB}=$ cardiopulmonary bypass \\ $\mathrm{ECMO}=$ extracorporeal membrane oxygenation \\ ICU = intensive care unit \\ LSCTS $=$ long segment congenital tracheal stenosis \\ OCT = optical coherence tomography \\ STP $=$ slide tracheoplasty}

arborization patterns, extent of tracheal stenosis, presence of bronchial stenosis, the need for mechanical ventilation before surgery, and the need for either pre- or postoperative extracorporeal membrane oxygenation (ECMO). Preoperative bronchomalacia was defined as dynamic collapse of the main bronchus by $>50 \%$ (by diameter) on biplane bronchography. Postoperative airway malacia was defined as dynamic collapse of the airway (either tracheal or bronchial) of $>50 \%$ diameter on biplane bronchography. Operative details and postoperative management, including complications and length of intensive care unit (ICU) stay and postoperative ventilation were recorded. The need for airway reintervention was defined as $\geq 3$ endoscopic dilations.

Tracheobronchial arborization patterns and extent of stenoses were defined as previously described. ${ }^{14}$ Arborization patterns were normal bifurcation, anomalous right upper lobe or porcine bronchus (bronchus suis), bronchial trifurcation, and single lung. Congenital bronchial stenosis was defined as the presence of complete tracheal rings in at least 1 bronchus (beyond carina or into a right upper lobe bronchus), with $>50 \%$ narrowing. ${ }^{14}$ Follow-up data, including the need for endoscopic interventional radiology procedures (eg, tracheal and/or bronchial dilation and/or stenting), were also collected.

\section{Patient Management}

All children referred to Great Ormond Street Hospital with LSCTS are channeled through a dedicated multidisciplinary tracheal team. Management follows a defined protocol and includes the following mandatory investigations: bronchoscopy and bronchography, echocardiography, and thoracic imaging with computed tomography and/or magnetic resonance imaging. Other investigations include lung volume-flow loops, optical coherence tomography (OCT), and cardiac catheterization when required.

STP is performed as previously described. ${ }^{1}$ Briefly, the surgery is performed through a median sternotomy with cardiopulmonary bypass (CPB). Where necessary, congenital cardiovascular anomalies are corrected at the same time. Before commencing $\mathrm{CPB}$, the anterior trachea is exposed and the entire length of stenosis identified by external inspection, or by needling the airway and identifying limits of the stenosis with flexible bronchoscopy. The trachea is completely mobilized to just beyond the limits of the complete rings, or until normal trachea and/or bronchi are seen. Further mobilization may occasionally be required, and can be achieved by releasing either Hilar lymph nodes below the carina or the posterior pericardial reflection inferiorly. Superior mobilization is routinely via division of the thyroid isthmus or very rarely by a hyoid release. If no cardiovascular repairs are required, normothermic CPB is established and the mobilized trachea divided at the midpoint of the stenosis. Longitudinal incisions are made on opposite sides of proximal (posteriorly) and distal (anteriorly) segments and the ends are spatulated. An oblique sliding anastomosis is performed with interrupted mattress polydioxanone (PDS) sutures. If a tracheostomy is present, and the stoma is adjacent to the stenosis we advocate it be incorporated into the repair, otherwise it is left for conventional de-cannulation once the patient needs only minimal ventilation. Assessment of the effectiveness of repair is judged by immediate postprocedural bronchoscopy and a leak test with gradual insufflation of the lungs with the repair submerged in warm saline. A mediastinal catheter is left in situ together with chest drains as required. Mediastinal irrigation with $1 \%$ povidone-iodine solution (reconstituted from $10 \%$ aqueous povidone iodine) in $0.9 \%$ saline is commenced at 2 $\mathrm{mL} / \mathrm{kg} / \mathrm{h}$ for 48 hours. Reservoir bags are changed every 24 hours and before catheter withdrawal samples are tested for microbial colonization (mediastinal contamination is assumed).

Postoperative management includes bedside fiberoptic bronchoscopy at 24 hours to plan extubation. Further evaluation of the airway is performed at 1 week with bronchoscopy and bronchography. Subsequent evaluations (with or without balloon dilations) are performed according to clinical need. Stent placement is only indicated in cases in which severe postoperative malacia prevents withdrawal of ventilatory support or in patients who fail to progress on noninvasive ventilation. ${ }^{1,13}$ In these children, a made-to-order biodegradable PDS stent (Ella-CS, Hradec Kralove, Czech Republic) is sometimes considered, because it resorbs over an 8- to 12-week period. ${ }^{15}$ Other stents previously inserted were balloon-expandable metal stents (Palmaz; Cordis, Warren, NJ), and self-expanding nitinol stents (Niti-S; Pyramed, Escher, UK).

Bronchoscopy and bronchography delineate the full anatomical configuration of the airway and provide dynamic information not easily achievable with other forms of imaging. As previously described, these procedures are performed simultaneously in an interventional radiology suite. ${ }^{16}$ The child is anesthetized with either a laryngeal mask airway or intubated, and without paralysis where possible. If intubated, the endotracheal tube is withdrawn so that the upper trachea may be examined. Small volumes $(<1 \mathrm{~mL})$ of iohexol (Omnipaque $240 \mathrm{mg} / \mathrm{mL}$; Nycomed Inc, Asker, Norway) are injected either via a catheter introduced through the endotracheal tube or through the working channel of the bronchoscope into the proximal trachea. Dynamic images are acquired in both posteroanterior and lateral projections. OCT is performed in select patients to clarify the status of airway cartilage formation. OCT is specifically used to determine if cartilage rings are complete, fragmented, or absent. Patients undergo OCT at the time of bronchoscopy and/or bronchography. A floppy-tipped 0.36-mm diameter OCT wire (ImageWire 200; St Jude Medical, St Paul, Minn) is passed through the side arm of the bronchoscope or the bronchographic catheter and advanced to the region of interest. Confirmation of positioning is obtained by direct visualization and/or bronchography. ${ }^{17,18}$ If balloon dilation is required; for example, for recurrent granulation tissue, an angioplasty catheter is selected (with a balloon approximately the same size as the normal airway) and inflated under fluoroscopic guidance until the stenosis is obliterated. Intervals between ballooning are determined by clinical symptoms and the appearance of the airway at bronchoscopy.

\section{Data Analysis}

Patient demographics and the nature of treatments were obtained from hospital records. Data are presented as mean values \pm standard deviation, medians and ranges, and/or binomial percentages, where appropriate. Kaplan-Meier survival curves were plotted for outcome measures and log-rank tests were performed to analyze differences between specific subgroups. Risk factors for mortality and stent insertion were analyzed with a Cox proportional-hazards regression model with the forward (likelihood ratio) method applied. Data were analyzed and displayed with SPSS software version 21.0 (IBM-SPSS, Inc, Armonk, NY) and Prism 6.0 (GraphPad Software Inc, La Jolla, Calif).

\section{RESULTS}

One hundred one children (median age, 5.8 months; range, 5 days-15 years) underwent STP. Patient demographics and general characteristics are shown in Table 1. All children had stenosis $>50 \%$ of the length of the trachea and $89 \%$ 
had $>80 \%$ involvement. Twenty-four patients $(24 \%)$ had a stenosis extending into the main bronchi. In 47 children $(47 \%)$, the STP was extended into the bronchus. Seventytwo patients $(71 \%)$ had additional cardiac or great vessel anomalies, 48 of whom (48\%) had a left pulmonary artery sling (Table 2). Cardiovascular anomalies were corrected during the same procedure. CPB was used in all cases and median time was 86 minutes (range, $38-323$ minutes). Three patients $(3 \%)$ had tracheotomies inserted by other units before referral. All 3 were decannulated at a later stage when ventilatory requirements were minimal. Surgery was not offered on 1 child with LSCTS due to the presence of severe congenital anomalies, including intractable severe bronchomalacia. During the period covered by our study, 9 children presented to our team with asymptomatic LSCTS and at the time the study was completed the patients were under surveillance without intervention. These patients are not included in our analysis.

A wide variety of airway arborization patterns ${ }^{14}$ were present in our cohort. Sixty-two patients $(61 \%)$ presented with normal tracheal bifurcation; 13 patients $(13 \%)$ with an anomalous right upper lobe bronchus; 17 patients $(17 \%)$ with tracheal trifurcation, and 9 patients $(9 \%)$ with a single lung. Forty-three children $(43 \%)$ children had concomitant congenital malformations. These included VACTERL/VATER association in 6 patients $(6 \%)$, anorectal malformations in 7 patients $(7 \%)$, and trisomy 21 in 8 patients $(8 \%)$. Preoperatively, 10 patients $(10 \%)$ required ECMO, whereas 56 patients $(55 \%)$ were mechanically ventilated. Only 35 children $(35 \%)$ were stable and self-ventilating in air before surgery. Twenty-four patients $(24 \%)$ had preoperative malacia as determined by bronchography. Median postoperative ventilation time was 11 days (range, $0-755$ days) and the median ICU stay was 20 days (range, 2-477 days).

TABLE 1. General characteristics and demographic data

\begin{tabular}{lc}
\hline \multicolumn{1}{c}{ Characteristic } & Result \\
\hline Age & 5.8 mo $(5 \mathrm{~d}-15 \mathrm{y})$ \\
Sex, male:female & $62: 39$ \\
Weight, kg & $5.9(1.9-38.3)$ \\
Trachea minimum diameter, mm & $2.2(1-4)$ \\
Preoperative ventilation & $56(55)$ \\
Preoperative bronchomalacia & $24(24)$ \\
Preoperative ECMO & $10(10)$ \\
Congenital bronchial stenosis & $24(24)$ \\
Abnormal arborization* & $39(39)$ \\
Cardiovascular association & $72(71)$ \\
Congenital malformations & $43(43)$ \\
Postoperative ventilation, d & $11(0-755)$ \\
Endoscopic reintervention post-STP & $48(48)$ \\
Postoperative airway malacia & $20(20)$ \\
\hline
\end{tabular}

Values are presented as median (range) or $\mathrm{n}(\%) . E C M O$, Extracorporeal membrane oxygenation; STP, slide tracheoplasty. ${ }^{*}$ Consisting of porcine bronchus, tracheal trifurcation, and single lung.
TABLE 2. Associated cardiovascular anomalies

\begin{tabular}{lr}
\hline Cardiovascular anomaly & $\mathbf{n}$ \\
\hline Ventricular septal defect & 15 \\
Atrium septal defect & 7 \\
Atrioventricular septal defect & 2 \\
Left pulmonary artery sling & 48 \\
Tetralogy of Fallot & 10 \\
Vascular ring & 4 \\
Subaortic stenosis & 3 \\
Truncus arteriosus & 2 \\
Hypoplastic aortic arch & 2 \\
Persistent ductus arteriosus & 4 \\
Right aortic arch & 2 \\
Other* & 5 \\
\hline
\end{tabular}

*Consisting of double outlet right ventricle $(\mathrm{n}=1)$, anomalous left subclavian artery $(\mathrm{n}=1)$, absent left pulmonary artery $(\mathrm{n}=1)$, aortopulmonary window $(\mathrm{n}=1)$, and right pulmonary artery hypoplasia $(\mathrm{n}=1)$.

Postoperative airway malacia (tracheal or bronchial) was present in 20 patients $(20 \%)$, but was not shown to be associated with either length of ICU stay $(P=.11)$ or duration of postoperative ventilation $(P=.07)$. Forty-eight children $(48 \%)$ required post-STP endoscopic intervention of the airway (balloon dilation and/or stenting) and 45 children $(45 \%)$ required balloon dilation (median 4 , range 1-27 times). Of those who underwent endoscopic ballooning, 33 children required $\geq 3$ dilations in a postoperative period of 4 months. Thirty-seven tracheal and/or bronchial stents were inserted into 22 patients $(22 \%)$ with 1 patient requiring 4 stents and another 7 stents (stent-in-stent). Stent insertion in the first decade was required in $29 \%$ (8 out of 29) of patients compared with $19 \%$ (14 out of 72 ) in the remainder of the study period. A variety of stents were used over this period, including $29(21 \%)$ balloon-expandable steel stents (Palmaz; Cordis), and $2(2 \%)$ Nitinol self-expandable stents (Niti-S; Pyramed). During the past 4 years there has been increasing trend to use bioabsorbable PDS stents (Ella-CS) which account for $85 \%$ of stents ( 6 out of 7 ) during this period. No tracheostomies were inserted during the study period.

Follow-up information was available for all patients for a median duration of 4.6 years (range, 2.7 months-17 years). In total there were 12 deaths $(12 \%)$ of which $6(6 \%)$ were early and $6(6 \%)$ were late. Four deaths were airway related, 4 cardiac related, and 4 due to other causes. To date there have been 5 deaths in the final 70 consecutive patients. Analysis comparing outcome measures between first and last quadrennial periods showed a significant difference in postoperative ventilation time with the median reducing from 67.5 days (range, 7-755 days) to 8.5 days (range, 1-109 days). (Figure 1).

Complications included 3 patients $(3 \%)$ with anastomotic dehiscence, of which 2 cases $(2 \%)$ were secondary to postoperative mediastinitis and required redo surgery (these occurred during a period when continuous suture 

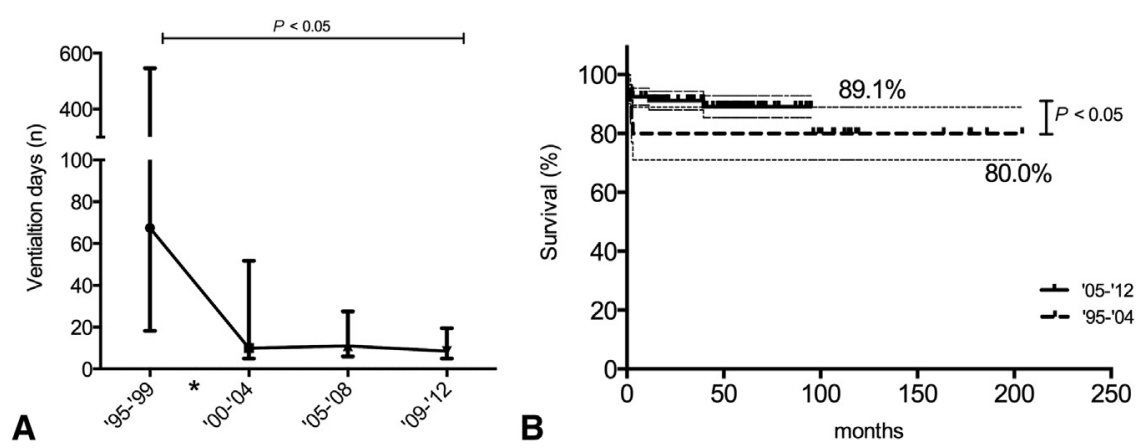

FIGURE 1. A, Acquired experience improves outcomes as demonstrated by reduction of postoperative ventilation days in serial quadrennial periods $(P<.05)$. Postoperative ventilation days are expressed as median and interquartile ranges. B, Actuarial survival demonstrating significant improvement in the second decade compared with the first $(P<.05)$. *Implementation of tracheal multidisciplinary team.

techniques were used). Thirty-three children $(33 \%)$ had residual stenosis at 3 months and $8(8 \%)$ had residual stenosis at 9 months. Six children $(6 \%)$ had persistent pneumothoraces requiring re-intervention, 4 children $(4 \%)$ had a chylothorax, 2 children $(2 \%)$ had pulmonary hypertension, and 4 children ( $4 \%$ ) had pneumopericardium or pneumomediastinum. Four children $(4 \%)$ had a phrenic nerve injury and 3 children ( $3 \%$ ) had a recurrent laryngeal nerve injury that spontaneously resolved within 6 months.

Variables included in multivariate analysis were age, sex, weight, minimal tracheal diameter, preoperative invasive ventilation, preoperative bronchomalacia, congenital bronchial stenosis, abnormal arborization of the airway, single lung, cardiovascular anomalies, other congenital anomalies, CPB time, postoperative malacia, and STP extending into the bronchus. Outcome measures for multivariate analysis were overall survival and freedom from stenting. Multivariate analysis revealed preoperative ECMO $(P<.05)$, congenital bronchial stenosis $(P<.05)$, and preoperative bronchomalacia $(P<.001)$ were predictors of mortality (Figure 2). Other variables were not significant for survival (Table 3). Multivariate analysis revealed preoperative malacia to be the only significant predictive indicator for stenting $(P<.05)$ (Figure 3$)$.

\section{DISCUSSION}

LSCTS typically has been associated with significant morbidity and mortality, with numerous reconstruction techniques advocated for repair of the airway. Since first being described in 1989 by Tsang and colleagues, ${ }^{19}$ STP has been adopted increasingly as the preferred surgical option for children with LSCTS, after early case series highlighted its efficacy. ${ }^{10,11}$ Its ease of application to a wide spectrum of stenotic airway disease has coined the phrase, "1 slide fits all," children with LSCTS but also extended to those with short segment stenosis and proximal tracheal disease. ${ }^{20-22}$

Although surgery in these children can be challenging due to their heterogeneous presentation, we have found the slide technique to be applicable to all airway morphologies with low morbidity and mortality. Previous studies have reported favorable outcomes for STP with mortality reported at $5 \%$ to $30 \%{ }^{4,7,10,23}$ However, these have been limited by either small cohort size and/or limited length of follow-up. The largest case series to date reported excellent outcomes with 5\% mortality in 80 children undergoing STP, but with a median follow-up of only 12 months. $^{7}$ Our mortality rate is higher, at $10 \%$, but this may reflect our initial learning curve, longer followup, and perhaps a more complex case mix. Certainly a greater proportion of our patients required preoperative ventilation and ECMO, and almost $25 \%$ had either congenital bronchial stenosis and/or bronchomalacia. Notably, such findings were either absent or unreported in the Cincinnati experience, ${ }^{7}$ suggesting genuine cohort differences between centers. Whereas this may simply reflect the heterogeneity of LSCTS, it could also be a result of differing referral pathways preselecting different patient cohorts. At Great Ormond Street Hospital for Children we see an unfiltered national population of patients, rather than a specifically referred population.

During the past 2 decades, our approach to managing children with LSCTS has naturally evolved, resulting in improvements in hospital stay, postoperative ventilation time, and survival. A significant learning curve was present in the first quadrennial period that dramatically improved with acquired experience, such that the final consecutive 70 patients had a mortality rate of $7 \%$. Although the application of STP in itself undoubtedly is a significant contributor to improved outcomes, we believe that the introduction of a multidisciplinary team has been central to sustained improvements. ${ }^{13}$

Complications have been far from trivial, but we have found a general improvement with increasing experience. Granulation tissue is a common complication of airway surgery, but we have observed that this is readily treated with serial balloon dilation and we adopted a proactive strategy toward treating this. With active surveillance 

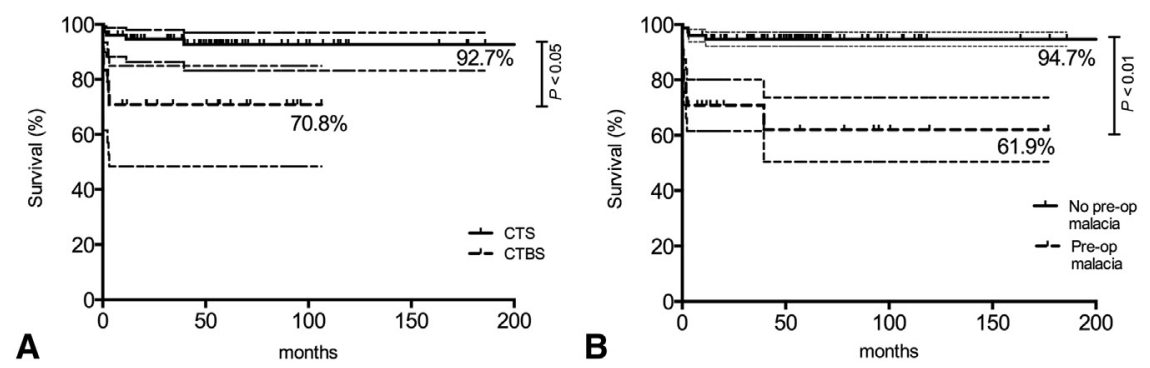

FIGURE 2. A, Actuarial survival in children with and without congenital bronchial stenosis (complete tracheal rings extending into the a main bronchus) $(P<.05)$. B, Survival for children with and without preoperative bronchomalacia $(P<.01)$. CTS, Congenital tracheal stenosis; $C T B S$, congenital tracheobronchial stenosis.

granulation tissue is treated with gentle ballooning before it becomes problematic. This might explain our higher intervention rates following surgery. Secondary (ie, acquired) stenosis is quite rare and the vast majority of patients $(82 \%)$ are intervention free within a year after surgery.

Predictors of adverse outcomes from our cohort have been identified as preoperative ECMO, preoperative bronchomalacia, and bronchial stenosis. It is unsurprising that the need for preoperative ECMO is an adverse predictor because it identifies a more challenging group. Typically, ECMO has been reserved for the "can't ventilate" scenario, and is likely to represent a cohort of more severe distal airway pathology. It is interesting to note the most adverse predictor in our cohort was preoperative distal malacia $(P<.001)$ where the hazard ratio was 8 . This can be quite problematic to treat because the STP does not always address this problem. In fact, a considerable amount of

TABLE 3. Multivariable regression* results with death or stenting as outcome variable

\begin{tabular}{|c|c|c|c|c|}
\hline \multirow[b]{2}{*}{ Characteristic } & \multicolumn{2}{|c|}{ Death } & \multicolumn{2}{|c|}{ Stenting } \\
\hline & $\begin{array}{c}\text { Hazard } \\
\text { ratio }\end{array}$ & $\begin{array}{c}P \\
\text { value }\end{array}$ & $\begin{array}{c}\text { Hazard } \\
\text { ratio }\end{array}$ & $\begin{array}{c}P \\
\text { value }\end{array}$ \\
\hline Age & & NS & & NS \\
\hline Sex & & NS & & NS \\
\hline Weight & & NS & & NS \\
\hline Minimum tracheal diameter & & NS & & NS \\
\hline Preoperative ventilation & & NS & & NS \\
\hline Bronchial stenosis & 4.7 & $<.05$ & & NS \\
\hline Preoperative malacia & 8.4 & $<.001$ & 3.0 & $<.05$ \\
\hline Preoperative ECMO & 5.4 & $<.05$ & & NS \\
\hline Cardiovascular association & & NS & & NS \\
\hline Congenital malformation & & NS & & NS \\
\hline CPB time & & NS & & NS \\
\hline Postoperative malacia & & NS & & NS \\
\hline Abnormal airway arborization & & NS & & NS \\
\hline Single lung & & NS & & NS \\
\hline Slide tracheoplasty into bronchus & & NS & & NS \\
\hline
\end{tabular}

$\overline{\text { Boldface indicates significant variables. } N S \text {, Not significant; } E C M O \text {, extracorporeal }}$ membrane oxygenation; $C P B$, cardiopulmonary bypass. *Cox regression model (forward [likelihood ratio] method applied). preoperative malacia may be masked due to inherent positive end-expiratory pressure generated by the stenosis. In our experience, distal malacia may only become clinically apparent when the stenosis is repaired by surgery. Because a notable proportion of our cohort was identified with preoperative malacia, it was presumed that longer postoperative ventilation and ICU stays may be a due to this phenomenon. Although our analysis did not reveal this to be a specific predictor it did trend toward significance $(P=.07)$. We believe this to be an important factor to define in the preoperative workup and we currently rely on bronchography to evaluate preoperative bronchomalacia. Our early experience with OCT assessment of the distal airway suggests that it is a very complimentary tool for evaluating the poor quality bronchial cartilage sometimes seen in children with LSCTS.

Stenting is sometimes considered for patients with severe recurrent stenosis (when serial balloon dilation fails and reoperation is not feasible) and/or malacia. Although the decision to stent is not taken lightly, it is sometimes unavoidable. The only factor that was a significant predictor of the need for stenting was preoperative bronchomalacia. Our own early experience with balloon expandable metal stents (Palmaz; Cordis) was not without complications. Stents readily colonize, promote excessive secretions, and are prone to secondary stenosis. ${ }^{24}$ As a result, we try to avoid stenting wherever possible. Despite this, we would in general prefer to insert $\geq 1$ stents than to perform tracheostomy following STP, especially now that bioabsorbable stents are available. ${ }^{15}$ Because postoperative airway malacia in most patients tend to resolve given enough time, noninvasive ventilation with continuous positive airway pressure is sometimes considered to ameliorate the situation while the airway stiffens.

Similar to others, we have found STP to be an extremely versatile technique that can be applied in patients with a variety of tracheobronchial arborization patterns. ${ }^{25,26}$ The ability to extend the slide is not only beneficial for bronchial stenosis but also for arborization patterns where there is a limited amount of trachea between the carina and an anomalous right upper lobe bronchus (porcine 

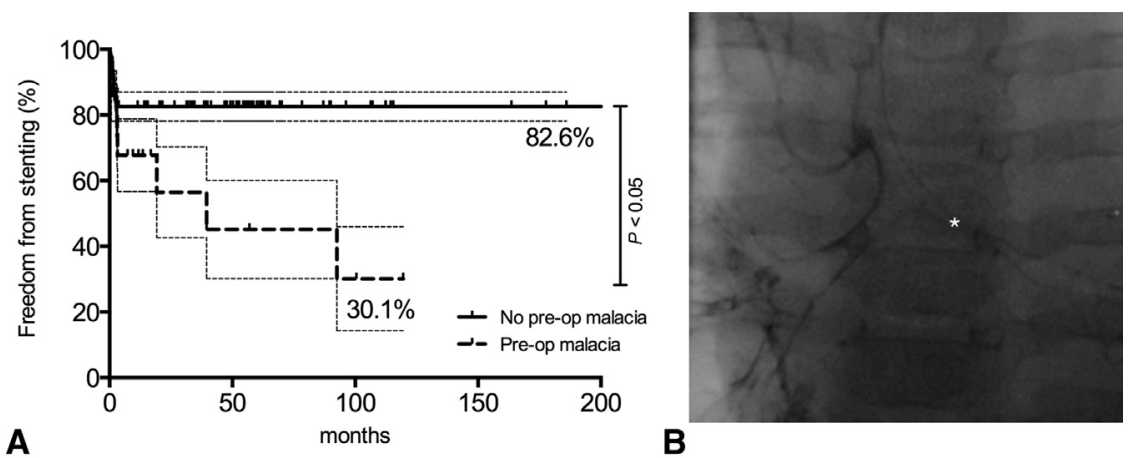

FIGURE 3. A, Kaplan-Meier plots demonstrating freedom from stenting for children with and without preoperative bronchomalacia $(P<.05)$. B, Preoperative malacia. *Bronchography shows $>50 \%$ diameter collapse in expiration in the left main bronchus.

bronchus). ${ }^{25}$ Understandably, extending the longitudinal incision into the bronchus is often met with some reservation, but it is noteworthy that the multivariate analysis did not reveal this to be a predictor of adverse outcome, suggesting the act of entering the bronchus, in itself, is not a risk factor. Instead, multivariate analysis showed bronchial stenosis itself to be more of a predictor of mortality. It is unclear what degree of stenosis would benefit from extending the slide repair, and it is possible that only the most stenotic bronchi need to be repaired, which would allow further reduction in morbidity where no clear benefit is to be gained. With this in mind (and similar to other groups) we now only opt to extend a slide only when absolutely necessary.

A limitation of our study is that the data are retrospective and despite being 1 of the largest reports, it is still relatively underpowered. Future studies will benefit from the data being prospective (and multicenter), and we welcome the formation an international registry for LSCTS. In our unit, a prospective database has been established ( 3 years), which will improve future reports. As the national referral center for complex pediatric tracheal disease, we manage almost all children in the United Kingdom with LSCTS, and we are confident that our cohort is representative of the range of patient characteristics found in LSCTS.

We acknowledge that our international referrals may alter our case mix by increasing the proportion of children with more complex disease, but this only accounts for a small percentage of cases $(11 \%)$ and has not influenced our analysis. Our study reports the longest follow-up to date, and it is very encouraging that most patients are in a stable condition 1 year after initial surgery. We are currently undertaking formal quality of life studies in our patient cohort, but we are comfortable reporting our impression that the vast majority of treated children are leading very good and largely asymptomatic lives.

\section{CONCLUSIONS}

STP is an extremely versatile technique that can be readily applied to the various airway configurations seen in LSCTS. Our data reveal predictors of adverse outcomes to be bronchial stenosis, preoperative ECMO, and preoperative malacia. We avoid stenting unless absolutely necessary, but when necessary bioabsorbable stents appear to be effective. Centralization of care has allowed us to gain considerable experience with this and other rare airway conditions, and we advocate such a strategic approach. We believe that a multidisciplinary team working with aggressive postoperative care is key to improved outcomes, and we consider STP to be the gold standard for LSCTS treatment, resulting in comparatively lower morbidity and mortality.

The authors thank the numerous members of the tracheal team both past and present, particularly Anja Fierens, Denise Mcintyre, Clare McLaren, Caroline Doyle, and Tabetha Tabiner, who have been essential to the efficient running of the Tracheal Team. The authors also thank previous Tracheal Fellows Hani Gabra and Michele Torre, the UK National Health Service Specialist Commissioning Team (formally NCG and AGNSS), and the patients and their families who so freely contributed their help and advice.

\section{References}

1. Elliott M, Hartley BE, Wallis C, Roebuck D. Slide tracheoplasty. Curr Opin Otolaryngol Head Neck Surg. 2008;16:75-82.

2. Elliott M, Roebuck D, Noctor C, McLaren C, Hartley B, Mok Q, et al. The management of congenital tracheal stenosis. Int J Pediatr Otorhinolaryngol. 2003;67(Suppl 1):S183-92.

3. Anton-Pacheco JL, Cano I, Comas J, Galletti L, Polo L, Garcia A, et al. Management of congenital tracheal stenosis in infancy. Eur J Cardiothorac Surg. 2006;29:991-6.

4. Chiu PP, Kim PC. Prognostic factors in the surgical treatment of congenital tracheal stenosis: a multicenter analysis of the literature. J Pediatr Surg. 2006; 41:221-5.

5. Herrera P, Caldarone C, Forte V, Campisi P, Holtby H, Chait P, et al. The current state of congenital tracheal stenosis. Pediatr Surg Int. 2007;23:1033-44.

6. Loeff DS, Filler RM, Vinograd I, Ein SH, Williams WG, Smith CR, et al. Congenital tracheal stenosis: a review of 22 patients from 1965 to 1987. J Pediatr Surg. 1988;23:744-8.

7. Manning PB, Rutter MJ, Lisec A, Gupta R, Marino BS. One slide fits all: the versatility of slide tracheoplasty with cardiopulmonary bypass support for airway reconstruction in children. J Thorac Cardiovasc Surg. 2011;141:155-61.

8. Valencia D, Overman D, Tibesar R, Lander T, Moga F, Sidman J. Surgical management of distal tracheal stenosis in children. Laryngoscope. 2011;121: 2665-71. 
9. Backer CL, Mavroudis C, Gerber ME, Holinger LD. Tracheal surgery in children: an 18-year review of four techniques. Eur J Cardiothorac Surg. 2001;19:777-84.

10. Grillo HC, Wright CD, Vlahakes GJ, MacGillivray TE. Management of congenital tracheal stenosis by means of slide tracheoplasty or resection and reconstruction, with long-term follow-up of growth after slide tracheoplasty. J Thorac Cardiovasc Surg. 2002;123:145-52.

11. Backer CL, Russell HM, Kaushal S, Rastatter JC, Rigsby CK, Holinger LD. Pulmonary artery sling: current results with cardiopulmonary bypass. J Thorac Cardiovasc Surg. 2012;143:144-51.

12. Grillo HC. Slide tracheoplasty for long-segment congenital tracheal stenosis. Ann Thorac Surg. 1994;58:613-9.

13. Kocyildirim E, Kanani M, Roebuck D, Wallis C, McLaren C, Noctor C, et al. Long-segment tracheal stenosis: slide tracheoplasty and a multidisciplinary approach improve outcomes and reduce costs. J Thorac Cardiovasc Surg. 2004; $128: 876-82$

14. Speggiorin S, Torre M, Roebuck DJ, McLaren CA, Elliott MJ. A new morphologic classification of congenital tracheobronchial stenosis. Ann Thorac Surg. 2012;93:958-61.

15. Vondrys D, Elliott MJ, McLaren CA, Noctor C, Roebuck DJ. First experience with biodegradable airway stents in children. Ann Thorac Surg. 2011:92:1870-4.

16. McLaren CA, Elliott MJ, Roebuck DJ. Tracheobronchial intervention in children. Eur J Radiol. 2005;53:22-34.

17. Torre M, Speggiorin S, Roebuck DJ, McLaren CA, Elliott MJ. Congenital absence of cartilaginous tracheal rings associated with esophageal atresia and trifurcated carina: a novel anomaly? J Pediatr Surg. 2012;47:1008-11.

18. Coxson HO, Eastwood PR, Williamson JP, Sin DD. Phenotyping airway disease with optical coherence tomography. Respirology. 2011;16:34-43.

19. Tsang V, Murday A, Gillbe C, Goldstraw P. Slide tracheoplasty for congenital funnel-shaped tracheal stenosis. Ann Thorac Surg. 1989;48:632-5.

20. de Alarcon A, Rutter MJ. Cervical slide tracheoplasty. Arch Otolaryngol Head Neck Surg. 2012;138:812-6.

21. Kim SM, Shin JH, Chang EY, Chang HK, Oh JT, Han SJ. Slide thyrocricotracheoplasty for the treatment of high-grade subglottic stenosis in children. J Pediatr Surg. 2010;45:2317-21.

22. Kim SM, Han SJ, Choi HS, Nam YT, Oh JT, Choi SH. Slide thyrocricotracheoplasty: a novel surgical technique for congenital laryngeal atresia. Pediatr Surg Int. 2008:24:383-6.

23. Matute JA, Romero R, Garcia-Casillas MA, de Agustin JC, Marhuenda C, Berchi FJ, et al. Surgical approach to funnel-shaped congenital tracheal stenosis. J Pediatr Surg. 2001;36:320-3.

24. Rijnberg FM, Butler CR, Speggiorin S, Fierens A, Wallis C, Nouraei SAR, et al. The influence of stents on microbial colonization of the airway in children after slide tracheoplasty: A 14-year single-center experience. Paediatr Pulmonol. 2014 [EPub ahead of print].

25. Beierlein W, Elliott MJ. Variations in the technique of slide tracheoplasty to repair complex forms of long-segment congenital tracheal stenoses. Ann Thorac Surg. 2006;82:1540-2.

26. Le Bret E, Roubertie F, Roger G, Sigal-Cinqualbre A, Coblence M, Belli E, et al. Slide tracheoplasty in infant with congenital tracheal stenosis and tracheomalacia after esophageal atresia with tracheoesophageal fistula repair. J Pediatr Surg. 2009; $44:$ e15-7.

\section{Discussion}

Dr Carl L. Backer (Chicago, Ill). First, I would like to acknowledge the efforts of Martin Elliott, who unfortunately could not be here today. Martin spearheaded use of the slide tracheoplasty and the emphasis on a multidisciplinary approach to these complex patients.

Dr Speggiorin, your experience is with more than 100 patients, $50 \%$ of them arriving intubated, and $10 \%$ arriving requiring extracorporeal membrane oxygenation. The mortality rate of only $7 \%$ in the final 70 patients demonstrates the excellent results that can be achieved with slide tracheoplasty and a focused team.
Our own series of tracheal stenosis operations at Lurie Children's Hospital now numbers more than 80 patients, and we have used the slide tracheoplasty technique in the past 18 patients. Our strategy change to use slide tracheoplasty instead of pericardial tracheoplasty and the autograft was largely based on the excellent results reported by your center, the late Dr Hermes Grillo, and Dr Peter Manning. Our experience shows that it is possible for a center that was heavily invested in 1 operationthe pericardial patch tracheoplasty was first performed at our institution-to change to a new technique (the slide tracheoplasty) based on the results of other surgeons.

It is interesting that you are using an interrupted suture technique that we originally proposed. We have abandoned interrupted sutures and changed to the running suture technique! We have also not used the betadine irrigation system in our 80 patients and have a very low incidence of mediastinitis I am hoping Dr Manning will comment on these 2 points.

I have 3 questions for you.

The first question is in regard to the use of simple tracheal resection and end-to-end anastomosis. When I first started my career, everyone said it was safe to resect $50 \%$ of the trachea Then a group from Boston proposed that you should resect no more than $30 \%$ of the trachea. I wonder if we should be using a slide tracheoplasty for almost all of these tracheal stenoses, even if it is a short segment of the trachea, say $10 \%$ to $20 \%$ of the total length. Do you have any comments about that?

Dr Speggiorin. I agree with you. When you have a short stenotic segment an end-to-end anastomosis is a good technique. Slide tracheoplasty can be used with both long and short stenotic segments. Theoretically, if you do a mini slide tracheoplasty on a short segment, the risk of postscarring stenosis can be minimized. It is the same principle that you can have when you do a coarctation repair. I know that the structures are different, but it is the same principle because the suture line is longer. Slide tracheoplasty also means shorter segments should be adopted.

Dr Backer. Thank you. I have had the experience of thinking I could do a tracheal resection and then suddenly realizing that the segment I need to resect is a lot longer than I thought and really wishing I would have just done a slide tracheoplasty.

My second question relates to 1 of the advantages to being in the United Kingdom. You use the phrase "quaternary referral center." It sounds like all of the patients in the United Kingdom with tracheal stenosis are coming to your single center. I guess you are probably going to agree with this, but the question is: Should surgeons who are only doing 1 tracheoplasty every couple of years be performing this operation or should they be referring patients to specialized centers that have a multidisciplinary team approach and extensive experience with this operation?

Dr Speggiorin. Following the principle that the more you do the better you become, I support this. Managing patients with very rare and complex disease can have a massive influence on the outcome if there is 1 institution where all the specialized care is centralized. Not only from the surgical point of view, because we realize that surgery is only a small part of patient care. The biggest part is what happens after surgery. This is shown by the fact that half of our patients need additional intervention after slide tracheoplasty. Having only 1 center within the entire United Kingdom-I know that is not possible in the United States 
because it is bigger-allows us follow-up with our patients in a very manageable way. We know where they are and can build a good relationship with the family. And the interventional radiologists are getting better as well. The more you do, the better you become, of course.

Dr Backer. My final question concerns the absorbable stent. I do not believe that is available in the United States at this point. We have had patients who have undergone slide tracheoplasty and then still have problems with bronchomalacia, particularly single-lung patients. Tell us about your experience with that stent. It looks like it worked pretty well.

Dr Speggiorin. Yes, the stent is a PDS stent. They are handcrafted in the Czech Republic. The initial experience was as an esophageal stent and then they started to put it in adult patients after lung transplant when they had a bronchial stenosis. We adopted these stents in kids.

The experience is not very big because we are dealing with a very rare situation, but we know that after a couple of monthsas it starts to dissolve-the stent starts to lose its radial force. It does not dissolve in 1 go; it is a slow process that gives us time to evaluate with serial bronchoscopy if the trachea becomes stiffer or not. So far we have had no technical problems in inserting these stents. The problem is that these stents are not on the market. You need to measure the airways and custom order a stent this length, this radial force, and this diameter. This is a little bit tricky sometimes, but we are using this device more and more.

Dr Peter Manning (St Louis, Mo). I have 2 comments and questions. But first I want to point out that the results of this experience are really remarkable. Some of the numbers from my own experience are not exactly comparable, because these authors report the kids with stenoses longer than $50 \%$, and a lot of reports include even shorter segment stenosis. You are to be congratulated for your results in this very complex end of the spectrum.

One comment and question I have is about the incidence of mechanical ventilation preoperatively. Half of the patients or more were on mechanical ventilators. I think in your univariate analysis that was predictive of mortality. Clearly, plastic tubes are not a friend of small airways. I think we all realize that. Most of these kids come to us intubated. But, frankly, most of these kids are not too symptomatic until a few months of age when they get that second hit-an upper respiratory infection-that trips them up and they wind up intubated. Then we have secondary damage. We give them inflammation and problems created by forcing an endotracheal tube into a small airway.

I think some of this morbidity is modifiable. We undertook an approach a number of years ago to try to get these kids extubated preoperatively. When I look back over our results, which did include all lengths of stenoses, $20 \%$ of the entire series of about 130 patients went to the operating room intubated. But when I look at just the least 50 patients, we had it down to $9 \%$. There were clearly a lot of factors leading to this, but some of it was related to an aggressive approach, of getting these kids extubated early to see if this is really a modifiable risk factor.
Have you had any experience trying to get these patients extubated, or off extracorporeal membrane oxygenation even, preoperatively? And what percent of kids came to you intubated and what percent ended up requiring intubation after arrival at your institution?

Dr Speggiorin. In our univariate analysis the preoperative ventilation was significant against death. When a patient is admitted to our institution and is intubated and ventilated, that patient is considered "urgent" and goes to the operating room using a preferential pathway. We do not consider extubating patients before surgery. Also, according to the multivariate analysis preoperative ventilation is not significant.

Dr Backer. In our series we rarely if ever go into the bronchus as part of our strategy for doing a slide tracheoplasty. One of the frequent telephone calls that I get is from a surgeon who is going to do a slide tracheoplasty saying that a radiologist has indicated that the patient has severe left bronchial stenosis, and what do I think about going into the bronchus? I almost always say that usually there is not an associated bronchial stenosis and often this is an overread by the computed tomography scan.

Now, in your series about $25 \%$ of patients had extension into the bronchus. It also seemed like those are the patients who got bronchomalacia and had problems with stents. So do you really think that is the right strategy?

Why don't you comment, and then Dr Manning can give us his analysis on this issue.

Dr Speggiorin. The indication for extending into the bronchus is whenever we find that there is a complete tracheal ring on the bronchus. Sometimes it can be found during the preoperative assessments and sometimes we find it once we are in the operative theater. When we see there is a stenosis, we just go inside.

On top of this, sometimes we use the bronchus for the repair because we extend the slide into the bronchus whenever we have a difficult anatomy. For example, when the right upper lobar bronchus takes off from the trachea. We use the slide to extend the slide into the bronchus to allow us to have a good slide.

Dr Backer. Peter, what are your thoughts? Do you go into the bronchus frequently?

Dr Manning. My experience mirrors yours, Carl, we haven't.

And my question is related to the evaluation. Bronchial stenosis is a difficult thing to evaluate. We did not have any experience with this using OCT or bronchography, but evaluating it endoscopically is difficult and dangerous. One probably should not be going down to the bronchus in these kids preoperatively with a bronchoscope. And computed tomography shows air in the airway. It cannot differentiate between stenosis and compression or even a mucous plug.

My question concerns these other diagnostic modalities, and did they supply additional information that prompted the decision to extend the repair into the bronchi? Extending into the bronchus may be creating more problems than you are solving. I have almost routinely limited the distal extent of the repair to the carina, even when the bronchus may look a little bit small on a computed tomography scan, and have not gotten into a lot of problems with the bronchi postoperatively by doing that. 